\title{
Análisis de la Gestión de las Tecnologias de la Información (TI) Dentro del Plan Estrategico Empresarial del Grupo Intercom
}

\author{
John Emmanuel Tobar Litardo, \\ Master en Sistemas de Información Gerencial, Ingeniero Comercial y \\ Empresarial, Coordinador de la carrera Tecnología Superior en Contabilidad, \\ Instituto Superior Tecnológico Juan Bautista Aguirre \\ Germán Edwin Frías Campos, \\ Master en Finanzas y Proyectos Corporativos, Economista con Mención en \\ Economía Internacional y Gestión en Comercio Exterior, Docente del \\ Instituto Superior Tecnológico Juan Bautista Aguirre \\ Darío Xavier Romero Santistevan, \\ Ingeniero en Electrónica y Telecomunicaciones, \\ Maestría en Ingeniería biomédica, Docentes del Instituto Superior \\ Tecnológico Juan Bautista Aguirre \\ Mildred Kenia Barrientos Alvarado, \\ Ingeniera en Ciencias Empresariales, Docente del Instituto Superior \\ Tecnológico Juan Bautista Aguirre
}

Doi:10.19044/esj.2019.v15n22p150 URL:http://dx.doi.org/10.19044/esj.2019.v15n22p150

\section{Resumen}

El presente estudio se centra en la necesidad de integrar los procesos bajo una planeación estratégica que considera la gestión de las TI como factor relevante en la competitividad del grupo INTERCOM. En función a lo planteado se propone analizar la percepción de los miembros del grupo empresarial basándose en la gestión de las tecnologías de la información (TI) para la integración de los diferentes procesos tanto operacionales como administrativos en función de la competitividad. Para el desarrollo del estudio se utilizó una metodología con un enfoque cualitativo que a través de la investigación no experimental, podría describir los factores relevantes contrastando las perspectivas estratégicas del Cuadro de Mando Integral con los recursos de las TI. De esta manera, se determinó la importancia de la gestión de las TI y la relación con la estrategia empresarial identificando un riesgo potencial de las TI en la perspectiva de aprendizaje por la falta de personal calificado que contribuye significativamente a la innovación, desarrollo y competitividad del grupo empresarial. 
Palabras claves: Gestión de TI, Plan estratégico, Balanced Scorecard, COBIT, Recursos de TI

\title{
Analysis of the Management of Information Technologies (IT) within the Business Strategic Plan of the Intercom Group
}

\author{
John Emmanuel Tobar Litardo,
}

Master en Sistemas de Información Gerencial, Ingeniero Comercial y Empresarial, Coordinador de la carrera Tecnología Superior en Contabilidad, Instituto Superior Tecnológico Juan Bautista Aguirre

Germán Edwin Frías Campos,

Master en Finanzas y Proyectos Corporativos, Economista con Mención en Economía Internacional y Gestión en Comercio Exterior, Docente del Instituto Superior Tecnológico Juan Bautista Aguirre

Darío Xavier Romero Santistevan, Ingeniero en Electrónica y Telecomunicaciones, Maestría en Ingeniería biomédica, Docentes del Instituto Superior Tecnológico Juan Bautista Aguirre

Mildred Kenia Barrientos Alvarado, Ingeniera en Ciencias Empresariales, Docente del Instituto Superior Tecnológico Juan Bautista Aguirre

Abstract

This paper focuses on the need to integrate the processes under a strategic planning that considers the management of Information Technologies (IT) as a relevant factor in the competitiveness of the INTERCOM group. Based on what has been proposed, we analyzed the perception of the members of the business group based on Information technology (IT) management. This is used for the integration of different operational processes which is both administrative and competitive. For the development of the study a methodology with a qualitative approach was used such that through nonexperimental research, the relevant factors could be described by contrasting the strategic perspectives of the Balanced Scorecard with IT resources. Therefore, the importance of IT management and the relationship with business strategy was determined. Also, a potential IT risk was identified in 
the learning perspective due to the lack of qualified personnel that contribute significantly to the innovation, development, and competitiveness of the business group.

Keywords: IT Management, Strategic Plan, Balanced Scorecard, COBIT, IT Resources

\section{Introducción}

En el desarrollo de cada una de las etapas dentro del proceso de direccionamiento estratégico es relevante la integración de los procesos y la comunicación de los miembros de la organización así como también de las áreas, departamentos, coordinaciones, etc. (Ibarra, Mballa, Ibarra, \& Vega, 2019) integrar determinados procesos es una labor difícil de generar sin contar con herramientas tecnológicas que aporten en la automatización de los mismos (Mahdzur \& Salim, 2015). Por tal motivo, la implementación de un sistema de información gerencial como instrumento que contribuya con el control del desempeño dentro de un plan estratégico es un aporte significativo para la toma de decisiones y la competitividad empresarial (Hernández, Cardona, \& Del Rio, 2017).

INTERCOM es un grupo empresarial que opera en zonas rurales en la costa ecuatoriana y está conformado por varios socios que distribuyen el servicio de Internet bajo un solo proveedor pero que además operan de forma independiente, es decir cada socio establece sus propias inversiones tanto en infraestructura tecnológica como en talento humano. En el 2019 decidieron por medio de una asamblea de socios integrar la organización desde el punto de vista operacional y administrativo. A pesar de que el grupo se operaba de forma independiente por parte de los socios, en la actualidad buscan presentar al grupo como una organización más sólida y competitiva.

En consecuencia, el presente estudio se plantea analizar la percepción de los miembros que operan en el grupo INTERCOM partiendo de la gestión de las tecnologías de la información (TI) como instrumento que integre los procesos en función de la competitividad del grupo empresarial.

\section{Marco referencial}

La gestión de las TI en América Latina ha evolucionado en cuanto a la economía digital la cual está constituida por medida de gastos en TIC, los usuarios de Internet y la diversificación de uso. Según la Comisión Económica para América Latina y el Caribe (CEPAL) (2013), esta región ha desarrollado un crecimiento durante la última década dicha región se ha transformado en un mercado emergente en el uso de aplicaciones TIC por parte del sector empresarial, del sector gubernamental y de los individuos alcanzando un gasto de 295000 millones de dólares en 2011 y cerca de 5,2\% del PIB (Gartner, 
2012). En el siguiente gráfico se representan los valores que componen la composición del gasto mundial por región y sector de aplicación:

- El gasto de América Latina fue equivalente a $8 \%$ del total mundial.

- Crecimiento sostenido que alcanzó un 12,1\% en 2011.

- El gasto mundial en TIC se descompone en telecomunicaciones (47\%), servicios TIC (24\%), dispositivos (17\%), software (8\%) y centros de datos $(4 \%)$.
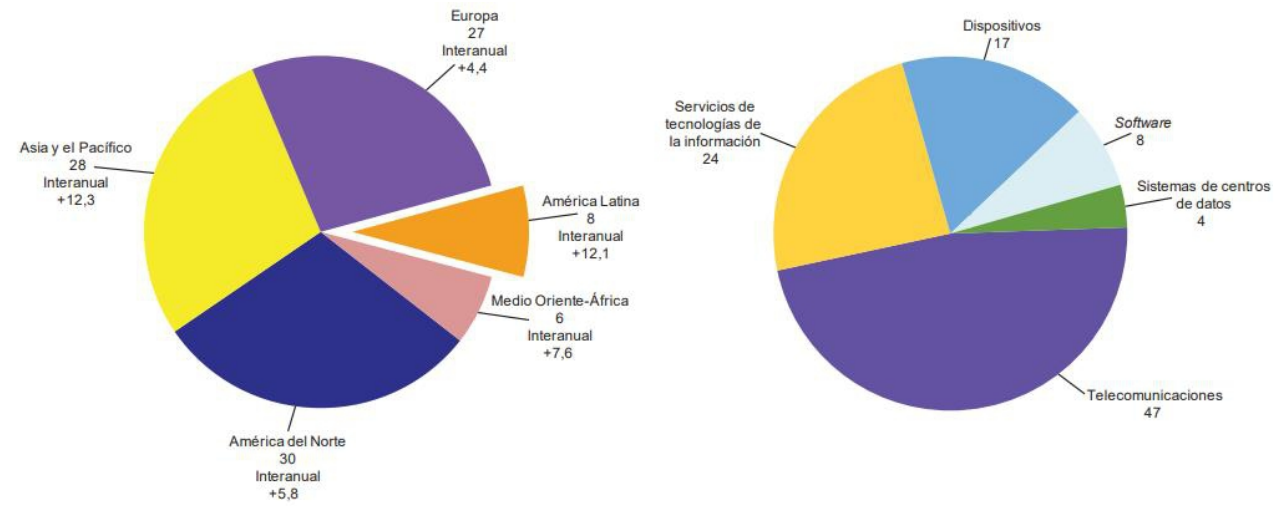

Figura 1. Composición del gasto mundial en TIC, según región y sector de aplicación, 2011 (en porcentajes)

Fuente: Comisión Económica para América Latina y el Caribe (CEPAL), sobre la base de Gartner Market Databook, 2012

En consecuencia, dichos ámbitos son analizados para que formen parte de una estructura base para el desarrollo de objetivos estratégicos dentro del plan a nivel organizacional, dicho plan también implica diferentes conjuntos de partes interesadas de los tomadores de decisiones tales como la parte interesada empresarial. Adaptarse a los cambios de procesos organizativos para resolver la gama de problemas del usuario podría llevar a cambios de estructura organizativa.

En Ecuador analizar el desarrollo de las Tecnologías de la Información (TI) significa la generación de un análisis retrospectivo en el cual se contrasta el actual desarrollo tecnológico del país en función del crecimiento económico relevante. Esto implica un escenario en el cual el tanto el sector empresarial como los consumidores inviertan en tecnología; sin embargo, anteriormente no pasaba (Moreira, Romero, Alava, Alcívar, \& Tarazona, 2018).

En concordancia con la clasificación de las regiones y la relación con el desarrollo de una matriz productiva es importante que tanto el sector público como privado cuenten con una herramienta imprescindible como la tecnología, porque este tipo de herramientas son capaces de integrar los procesos en función del plan estratégico que garantice el empleo de las TI a largo plazo y, por lo tanto, el fortalecimiento de manera directa del tanto del sector estratégico como también de la independencia tecnológica, 
contribuyendo no sólo a la seguridad tecnológica sino también a la adaptación a las situaciones socioeconómicas propias del Ecuador.

Los avances generados luego de implementar la tecnología como instrumento dan lugar a la innovación. Este producto final representa uno de los recursos que logra mayores ventajas competitivas y como estrategia empresarial fomenta un mayor nivel de crecimiento como también de desempeño (Keupp, Palmié, \& Gassmann, 2012). Por otra parte, Ibujés y Franco (2019) plantean un pronóstico en relación a innovación donde se genera una inversión de cerca del 2\% del PIB en Actividades de Ciencia, Tecnología e Innovación (ACTI) para el año 2019 donde se presenta una leve tendencia al crecimiento. Según el ranking global Innovation Index 2018, Ecuador apenas llega con un puntaje de 26,8 sobre 100 en innovación, alcanzando el puesto $97^{\circ}$ de entre 126 economías en el mundo, cinco puestos más abajo que en el año 2017 (Dutta, Lanvin, \& Wunsch, 2018). Esto indica que es inevitable el desarrollo urgente de propuestas para mejorar los procesos de innovación en los diferentes sectores de la economía con perseverancia en el planteamiento de políticas y planes estructurales que amplíen la infraestructura de las TIC para fortalecer el uso de las mismas y el desarrollo de habilidades digitales en todos los ciudadanos.

Moon et al. (1995), determinan un nuevo paradigma de competitividad empresarial al considerar la apertura de fronteras para ser partícipe en la economía mundial, así como también para impulsar los mercados locales. La característica de este tipo de competitividad sistémica integra tanto al sector empresarial como la industria, el gobierno y la población de un país. Estas acciones estratégicas promueven la cooperación o alianzas estratégicas entre competidores, clientes y proveedores. Como resultado de esta integración se obtiene confianza, además del logro de generar ventajas competitivas, haciendo posible la reestructuración del valor empresarial desde el punto de vista financiero (Arredondo, De la Garza, \& Villa, 2015).

Buele et al. (2019) determinan que para empresas en las cuales la preservación y confidencialidad de la información es una prioridad puesto que esta fomenta la continuidad de la misma, la generación de objetivos de control (Ver tabla 1) enfocados a las perspectivas estratégicas de los procesos internos y que son determinados bajo el modelo COBIT, puede establecer una mejoría significativa en cada paso que comprenden los procesos a mediano plazo. Sin embargo, se establece que cada objetivo de control esté relacionado con uno o más procesos de las TI; de esta forma se pretenden integrar los procesos, así como también los dominios del modelo y como resultado se logrará entrelazar varios aspectos que podrían identificarse en tiempos prudenciales sin dejar a un lado la vulnerabilidad que afecte a la organización. 
Tabla 1. Objetivo de control - Planear y Organizar

\begin{tabular}{|l|l|}
\hline Objetivo de control & Descripción \\
\hline PO1 & Definir el plan estratégico de TI. \\
\hline PO2 & Definir la arquitectura de la información \\
\hline PO3 & Determinar la dirección tecnológica. \\
\hline PO4 & Definir procesos, organización y relaciones de TI. \\
\hline PO5 & Administrar la inversión en TI. \\
\hline PO6 & Comunicar las aspiraciones y la dirección de la gerencia. \\
\hline PO7 & Administrar recursos humanos de TI. \\
\hline PO8 & Administrar calidad. \\
\hline PO9 & Evaluar y administrar riesgos de TI \\
\hline PO10 & Administrar proyectos. \\
\hline PO11 & Administración de Calidad \\
\hline
\end{tabular}

Fuente: (Buele y otros, 2019)

Para la integración de los procesos que conforman la gestión de las TI es relevante conformar un comité informático, liderado por quien tenga la responsabilidad de la planeación estratégica en calidad de director además de realizar las evaluaciones de desempeño en los procesos de servicios y de los recursos tecnológicos. De esta manera se genera la creación de la cultura de las TI a partir de las cuales se relacionen y adapten cambios de esta estructura, incorporando la adquisición de nuevos conceptos, modelos, procesos, gestión de conocimiento, trabajo en equipo y gestión del cambio (Andrade \& Morales, 2019).

En concordancia con la adaptación a los cambios estructurales dentro de una organización, la tecnología es una herramienta útil para la integración de procesos ya que esta permite medir el desempeño de objetivos estratégicos. El modelo IT4+ tiene como objetivo analizar la aplicación de la tecnología en función al mejoramiento de la gestión, fomentando que los procesos alcancen una mayor eficiencia y transparencia en la ejecución, una mejora en el acceso al proceso administrativo, y permitiendo el control de los recursos para brindar información objetiva y en el momento oportuno. Estas características permiten la toma de decisiones en todos los niveles (Marulanda, Valencia, \& López, 2017).

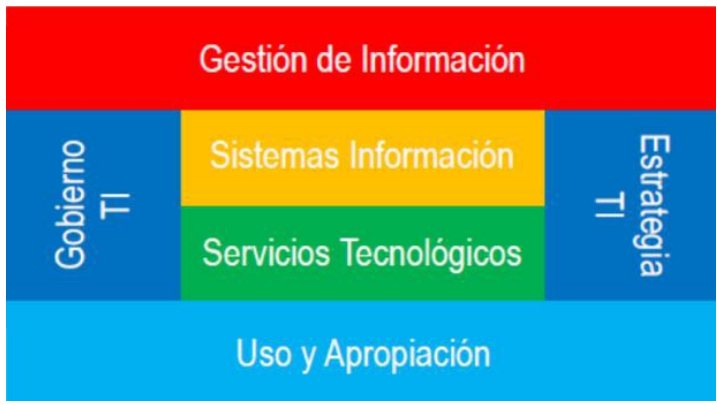

Figura 2. Modelo IT4+

Fuente: (Gómez, Valencia, \& Marulanda, 2018) 
Este modelo alinea la gestión de las TI con los objetivos estratégicos de la organización, el cual impulsa la eficiencia y mejora la calidad de los servicios o productos finales. El IT4+ mejora las condiciones de acceso a una gestión de las TI, haciendo un aporte significativo al valor estratégico desde el punto de vista de las necesidades del sector, la entidad, clientes y usuarios. Como se muestra en la figura 3, el modelo determina los siguientes componentes:

- Estrategia de TI

- Gobierno de TI

- Análisis de información

- Sistemas de información

- Gestión de servicios tecnológicos

- Apropiación y uso

Para la gestión de las TI, la normativa COBIT 5.0 da lugar al análisis de las TI en función a la forma de ser gestionadas bajo un enfoque holístico y ella incide en toda la estructura organizacional, de tal forma que se integran las funciones de áreas y responsabilidad de las TI de inicio a fin así como también aquellas internas y externas. Esta normativa se caracteriza por ser genérica y aplicable a organizaciones de todos los tamaños, tanto privadas como públicas (Velásquez, Pérez, \& Flórez, 2018).

La COBIT 5.0 se desarrolla y fundamenta bajo cinco principios claves para la gestión de las TI empresariales, estos principios son: satisfacer las necesidades de las partes interesadas, cubrir la empresa extremo-a-extremo, aplicar un marco de referencia único integrado, hacer posible un enfoque holístico y separar el gobierno de la gestión (Cobit 5.0. ISACA, 2012).

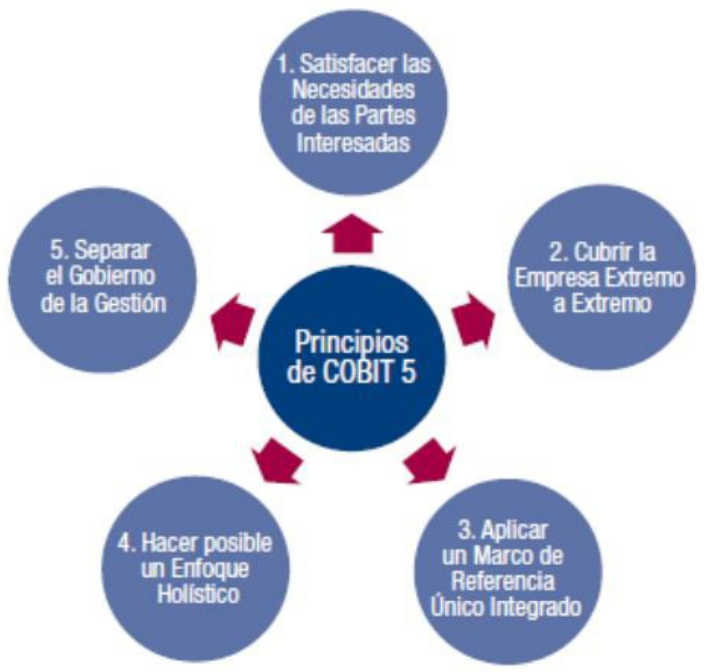

Figura 3. Principios de COBIT 5

Fuente: (Cobit 5.0. ISACA, 2012) 
Según Huamán (2014), para la empresa que se determine a priorizar y seleccionar instrumentos de medición capaces de identificar como también controlar los riesgos en la gestión de las TI, la norma de calidad ISO 27001 de las Normas internacionales de la Organización Internacional de Estandarización (International Organization for Standardization (ISO) es una opción para dicha acción, debido a que se analizan y gestionan los riesgos dentro de una planificación contribuyendo de forma significativa a la revisión y corrección para alcanzar la mejora continua de las TI implementadas en la organización con otros sistemas de gestión. La seguridad de la información según la ISO 27001 se enfoca en hacer prevalecer la confidencialidad, integridad y disponibilidad, así como también los diferentes sistemas de gestión (López, 2014). Esta normativa enmarca una serie de cláusulas que conforman un Sistema de Gestión de Seguridad de la Información conformada por:

- Liderazgo y compromiso

- Política

- Roles, responsabilidades y autoridades en la organización

Según Hidalgo et al. (2018) la metodología de análisis forense informático permite aumentar la efectividad y fiabilidad en los procesos de análisis en función del valor potencial de la información obtenida como el coste asociado a su obtención. Por lo tanto la ISO/IEC 27001 describe la Seguridad de la Información como una preservación de la confidencialidad, integridad y disponibilidad de la misma, en congruencia con la COBIT 5 la cual describe la gestión de las TI como un objetivo que garantiza el desarrollo de las tecnologías de información en función de la estrategia de la empresa y el alcance progresivo de las metas corporativas. Además de garantizar la funcionalidad de las TI, la ISO resalta las capacidades de las TI enfocadas a la efectividad y eficiencia, como se puede ver en la siguiente tabla (Castro, Velásquez, \& Castro, 2018).

Tabla 2. Análisis comparativo de COBIT - ISO 27001

\begin{tabular}{|l|l|l|l|l|}
\hline \multicolumn{2}{|l|}{ COBIT 5.0 } & ISO 27001 \\
\hline APO02 & $\begin{array}{l}\text { Gestionar la } \\
\text { Seguridad }\end{array}$ & Requerimiento & Cobertura & Justificación \\
\hline APO02.01 & $\begin{array}{l}\text { Entender la } \\
\text { dirección de la } \\
\text { empresa }\end{array}$ & $\begin{array}{l}\text { 4.2) } \\
\text { Comprenderlas } \\
\text { necesidades y } \\
\text { expectativas de } \\
\text { las partes } \\
\text { interesadas }\end{array}$ & A+ & $\begin{array}{l}\text { La ISO indica que se } \\
\text { tienen que } \\
\text { comprender las } \\
\text { necesidades de las } \\
\text { partes interesadas y } \\
\text { estas deberán tener } \\
\text { acceso a la } \\
\text { información } \\
\text { pertinente. }\end{array}$ \\
\hline
\end{tabular}




\begin{tabular}{|c|c|c|c|c|}
\hline APO02.02 & $\begin{array}{l}\text { Evaluar el } \\
\text { entorno actual, } \\
\text { las capacidades } \\
\text { y el rendimiento }\end{array}$ & $\begin{array}{l}\text { 5.3) Roles } \\
\text { organizacionales, } \\
\text { responsabilidades } \\
\text { y autoridades }\end{array}$ & $\mathrm{A}+$ & $\begin{array}{l}\text { La ISO nos indica } \\
\text { que cada persona } \\
\text { deberá tener la } \\
\text { capacidad de asignar } \\
\text { roles y } \\
\text { responsabilidades de } \\
\text { acuerdo a su } \\
\text { capacidad } \\
\end{array}$ \\
\hline APO02.03 & $\begin{array}{l}\text { Definir las } \\
\text { capacidades de } \\
\text { TI las y sus } \\
\text { objetivos }\end{array}$ & $\begin{array}{l}\text { 6.1) Acciones } \\
\text { para dirigir los } \\
\text { riesgos y } \\
\text { oportunidades, } \\
6.1 .2 \text { Evaluación } \\
\text { de riesgos de } \\
\text { seguridad de } \\
\text { información }\end{array}$ & A- & $\begin{array}{l}\text { La ISO nos indica } \\
\text { que se deben evaluar } \\
\text { los riesgos y las } \\
\text { oportunidades parar } \\
\text { guiar el } \\
\text { direccionamiento del } \\
\text { negocio }\end{array}$ \\
\hline APO02.04 & $\begin{array}{l}\text { Conducir un } \\
\text { análisis GAP }\end{array}$ & $\begin{array}{l}\text { No se puede } \\
\text { mapear }\end{array}$ & (N/A) & No aplica \\
\hline APO02.05 & $\begin{array}{l}\text { Definir el plan } \\
\text { estratégico y el } \\
\text { plan de } \\
\text { proyectos y } \\
\text { objetivos a } \\
\text { seguir }\end{array}$ & 5) Liderazgo & A & $\begin{array}{l}\text { La ISO nos indica } \\
\text { que un manager de } \\
\text { alto nivel debe } \\
\text { asumir un liderazgo y } \\
\text { un compromiso con } \\
\text { los objetivos de la } \\
\text { organización. }\end{array}$ \\
\hline APO02.06 & $\begin{array}{l}\text { Comunicar la } \\
\text { estrategia y la } \\
\text { dirección de las } \\
\text { TI }\end{array}$ & $\begin{array}{l}\text { 7.4) } \\
\text { Comunicación }\end{array}$ & A & $\begin{array}{l}\text { La ISO nos indica } \\
\text { que se debe } \\
\text { comunicar entre } \\
\text { todas la partes } \\
\text { interesadas }\end{array}$ \\
\hline
\end{tabular}

Fuente: PUCP (http://textos.pucp.edu.pe/pdf/3560.pdf)

En función al desarrollo del marco referencial, el modelo que se acopla a los objetivos del estudio está basado en la estructura de gestión de las TI que describe la COBIT, debido a que dicho modelo se ajusta a planes estratégicos empresariales que buscan por medio de las tecnologías de la información integrar los procesos para alcanzar los objetivos trazados por el grupo INTERCOM como lo es la competitividad.

\section{Metodología}

Enfoque

El presente estudio se desarrolló bajo un enfoque cualitativo en función del análisis conceptual de normativas o modelos que influyen en la gestión de TI y que se alinean como también infieren en la planificación estratégica. 


\section{Diseño}

Se estableció un estudio de diseño no experimental en el cual por medio de un alcance descriptivo se analizaron los factores de la gestión de las TI en función de la situación actual del plan estratégico del grupo empresarial.

\section{Unidad de análisis}

Como unidad de análisis se estableció proporcionar información a los 10 (diez) gerentes propietarios de empresas proveedoras de Internet que conforman el grupo INTERCOM en Ecuador. Esta organización se enfoca en prestar servicios de telecomunicaciones, principalmente en proveer servicio de internet a zonas rurales, donde otras operadoras no llegan, de esta forma aprovecha la concentración de la competencia.

\section{Técnica e instrumento de recolección de información}

La técnica e instrumento de recolección de información utilizada fue la encuesta. Para generar el diseño se adaptó el Modelo EFQM de Excelencia (Brume, 2017) que tiene como objetivo analizar el funcionamiento de los sistemas de gestión de una organización, en este proceso se van a clasificar los ítems de la encuesta en dos (2) categorías de análisis como son: gestión estratégica y herramientas para promover la competitividad. De estas se desprenden seis (6) dimensiones, como se puede apreciar en la siguiente figura:

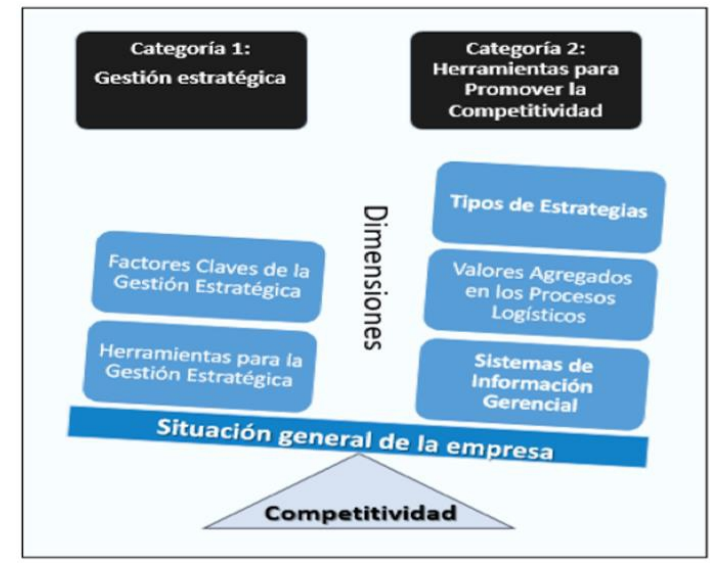

Figura 4. Categorías y dimensiones

Fuente: (Brume, 2017)

Elaborado por: El Autor

\section{Resultados}

Para el desarrollo de la encuesta se estructuró un análisis del diseño, partiendo de la herramienta de planeación estratégica basada en la COBIT como lo es el Balanced Scorecard (Cuadro de Mando Integral) desarrollada por los catedráticos de Harvard Kaplan y Norton en los 90’s (1992). Este 
instrumento divide el plan estratégico y la organización en cuatro perspectivas estratégicas (Financiera, Clientes, Procesos y Aprendizaje). Estas perspectivas se contrastaron con los cuatro recursos de las TI que se implementan en la gestión de TI y están alineadas a la estrategia de la organización.

En congruencia con el análisis se procedió a realizar una encuesta, en la cual los entrevistados (10 Gerentes del grupo INTERCOM-Ecuador) valoraron cada recurso en cada perspectiva; de esta forma se buscó identificar cuáles son los factores relevantes relacionados entre la gestión de las TI y la planeación estratégica como se puede apreciar en la siguiente tabla.

Tabla 2. Recursos de TI-Perspectiva financiera

\begin{tabular}{|l|l|l|}
\hline \multirow{2}{*}{ Herramientas para promover la competitividad } & \multicolumn{2}{l}{$\begin{array}{l}\text { Gestión Estratégica } \\
\text { Perspectiva } \\
\text { Financiera }\end{array}$} \\
\cline { 2 - 3 } & SI incide & NO incide \\
\hline Existen aplicaciones & $82,50 \%$ & $17,50 \%$ \\
\hline Se gestiona información & $82,50 \%$ & $17,50 \%$ \\
\hline Se cuenta con infraestructura tecnológica & $85,00 \%$ & $15,00 \%$ \\
\hline Se cuenta con personal capacitado & $82,50 \%$ & $17,50 \%$ \\
\hline
\end{tabular}

Elaborado por: Los autores con fuente de (INTERCOM-Ecuador)

Este análisis muestra que el grupo INTERCOM presenta una incidencia superior al $80 \%$ en los cuatro tipos de recursos de las TI dentro de la perspectiva financiera; esto quiere decir que los procesos financieros como ventas, utilidades, flujos, depreciaciones e impuestos se generan dentro de una plataforma virtual cuya infraestructura tecnológica es capaz de soportar y donde el personal que opera dichos procesos es relativamente competente en lo que respecta a la tecnología implementada hasta el momento.

Tabla 3. Recursos de TI-Perspectiva clientes

\begin{tabular}{|l|l|l|}
\hline \multirow{2}{*}{ Herramientas para promover la competitividad } & & \multicolumn{2}{|l|}{ Gestión Estratégica } \\
\cline { 3 - 4 } & Perspectiva de clientes \\
\cline { 3 - 4 } & SI incide & NO incide \\
\hline Existen aplicaciones & $65 \%$ & $35 \%$ \\
\hline Se gestiona información & $82 \%$ & $18 \%$ \\
\cline { 1 - 1 } Se cuenta con infraestructura tecnológica & $75 \%$ & $25 \%$ \\
\hline Se cuenta con personal capacitado & $67 \%$ & $33 \%$ \\
\hline
\end{tabular}

Elaborado por: Los Autores con fuente de (INTERCOM-Ecuador)

En la perspectiva de los clientes, existen inconvenientes en la implementación de recursos de las TI como las aplicaciones, las cuales permitirían a las empresas que conforman el grupo contar con un canal de comunicación externa con los clientes para evaluar el servicio prestado y promocionar la imagen corporativa. Sin embargo, otro factor está enfocado en el personal, puesto que varias empresas del grupo al no contar con aplicaciones 
para este tipo de servicio dirigido a los clientes, tampoco pueden contratar personal que se enfoque en dicha actividad.

Tabla 4. Recursos de TI - Perspectiva de procesos

\begin{tabular}{|c|c|c|}
\hline \multirow{2}{*}{ Herramientas para promover la competitividad } & \multicolumn{2}{|c|}{$\begin{array}{c}\text { Gestión Estratégica } \\
\text { Perspectiva de procesos }\end{array}$} \\
\cline { 2 - 3 } & SI incide & NO incide \\
\hline Existen aplicaciones & $82 \%$ & $18 \%$ \\
\hline Se gestiona información & $82 \%$ & $18 \%$ \\
\hline Se cuenta con infraestructura tecnológica & $80 \%$ & $20 \%$ \\
\hline Se cuenta con personal capacitado & $82 \%$ & $18 \%$ \\
\hline
\end{tabular}

Elaborado por: Los autores con fuente de (INTERCOM-Ecuador)

Los procesos internos del grupo INTERCOM muestran un soporte promedio del $82 \%$ en la implementación de todos los recursos de las TI enfocados en la planeación estratégica, este resultado demuestra que cuenta con procesos operacionales monitoreados por sistemas de información gerencial, debido también a la necesidad de proveer el servicio de Internet en zonas rurales alejadas y a las operaciones de instalaciones de antenas y redes de fibra óptica que se realizan en zonas de difícil acceso.

Tabla 5. Recursos de TI - Perspectiva de procesos

\begin{tabular}{|c|c|c|}
\hline \multirow{3}{*}{$\begin{array}{l}\text { Herramientas para promover la } \\
\text { competitividad }\end{array}$} & \multicolumn{2}{|c|}{ Gestión $\quad$ Estratégica } \\
\hline & \multicolumn{2}{|c|}{ Perspectiva de aprendizaje } \\
\hline & SI incide & NO incide \\
\hline Existen aplicaciones & $50 \%$ & $50 \%$ \\
\hline Se gestiona información & $37 \%$ & $63 \%$ \\
\hline Se cuenta con infraestructura tecnológica & $28 \%$ & $72 \%$ \\
\hline Se cuenta con personal capacitado & $37 \%$ & $63 \%$ \\
\hline
\end{tabular}

Elaborado por: Los Autores con fuente de (INTERCOM-Ecuador)

En la perspectiva de aprendizaje se encontraron factores más relevantes del análisis, debido a que las empresas presentan problemas enfocados en la falta de personal calificado específicamente ingenieros en sistemas, redes, programadores, técnicos en telecomunicaciones, etc. La problemática se basa a que los servicios de Internet se prestan en zonas rurales, donde tanto la concentración de profesionales como de universidades es escasa, por lo que para las empresas es difícil conseguir personal con dicho perfil, provocando un problema de importancia que repercute en la calidad del servicio y el cumplimiento de los objetivos estratégicos.

\section{Conclusiones}

El análisis de los factores que corresponde a la percepción de los diez miembros que operan en el grupo INTERCOM, demostró la importancia de la implementación de herramientas tecnológicas en el control del desempeño de 
los procesos, partiendo de bases conceptuales como las que describen las normativas COBIT en contraste con instrumentos estratégicos como el cuadro de mando integral de Norton y Kaplan y la incidencia que tiene bajo las cuatro perspectivas estratégicas basadas en la competitividad.

Por lo tanto, la presente investigación describe la importancia de la gestión de los procesos basados en un instrumento estratégico que se plantea bajo cuatro perspectivas estratégicas tales como: financiera, clientes, procesos internos y aprendizaje. Las cuatro perspectivas estratégicas se dinamizan de forma transversal proponiendo una integración (causa y efecto) entre los objetivos estratégicos y las acciones necesarias para su cumplimiento, es por este motivo que el estudio se propone describir la evaluación de los procesos por medio de un sistema de información gerencial cuya gestión de TI mida el desempeño de los procesos y el cumplimiento de los objetivos estratégicos para la toma de decisiones.

Según los resultados obtenidos, la perspectiva que tuvo un mayor impacto es la perspectiva de aprendizaje, esto indica que existe una debilidad y que puede ser identificada como un riesgo de TI potencial para la competitividad del grupo INTERCOM, debido a la falta de personal calificado que sea capaz de gestionar procesos técnicos enfocados al ensamblaje y mantenimiento de equipos tecnológicos, así como también que tenga experiencia con sistemas de información gerencial.

\section{References:}

1. Andrade, T., \& Morales, J. (2019). GOBIERNO TI APLICANDO ESTANDARES Y UN MARCO DE CONTROL EN EL USO DE COBIT 5 - ISO/IEC 38500. ESCUELA SUPERIOR POLITÉCNICA AGROPECUARIA DE MANABÍ MANUEL FÉLIX LÓPEZ.

2. Arredondo, F., De la Garza, J., \& Villa, L. (2015). Colaboración organizacional desde la perspectiva del empleado: caso de las maquiladoras del noreste de México. Revista Venezolana de Gerencia, 698-716.

3. Brume, M. (2017). Gestión estratégica como herramienta para promover la competitividad de las empresas del sector logístico del departamento del Atlántico, Colombia. Espacios, Vol. 38 ( $\left.\mathrm{N}^{\circ} 51\right)$.

4. Buele, J., Espinoza, J., Fiallos, D., Ruiz, M., Pilco, A., Reyes, J., \& Salazar, F. (2019). Aplicación del modelo COBIT para la administración de datos (DS11): el caso de un centro de análisis clínico. RISTI - Revista Ibérica de Sistemas e Tecnologias de Informação, 1010-1021.

5. Castro, D., Velásquez, T., \& Castro, H. (2018). Integración de seguridad y gestión de servicios en el gobierno de las tecnologías de la 
información. Revista Colombiana de Tecnologías de Avanzada (RCTA), 62-67.

6. CEPAL (2013). Economía digital para el cambio estructural y la igualdad. Naciones Unidas.

7. Cobit 5.0. ISACA. (2012). A Business Framework for the Governance and Management of Enterprise IT.

8. Dutta, S., Lanvin, B., \& Wunsch, S. (2018). Global Innovation Index 2018: Energizing the World with Innovation. Recuperado a partir de http://www.amazon.com/Global-Innovation-01-02-Express-

Exec/dp/184112219X/ref=sr_1_40?s=books\&ie=UTF8\&qid=13752 $04263 \& s r=1-40 \&$ keywords $=\% 22$ participative + design $+\% 22$.

9. Gartner. (2012). Gartner Market Databook. tercer trimestre.

10. Gómez, C., Valencia, F., \& Marulanda, C. (2018). Las Tecnologías de la Información y las Comunicaciones y los Servicios Tecnológicos en las Entidades Públicas del Triángulo del Café en Colombia. Información Tecnológica, 119-126. doi:http://dx.doi.org/10.4067/S0718-07642018000400119

11. Hernández, H., Cardona, D., \& Del Rio, J. (2017). Direccionamiento Estratégico: Proyección de la Innovación Tecnológica y Gestión Administrativa en las Pequeñas Empresas. Información tecnológica, 28(5), 15-22. doi:doi: 10.4067/S0718-07642017000500003

12. Hidalgo, I., Yasaca, S., Hidalgo, B., Oquendo, V., \& Salazar. (2018). Estudio Comparativo De Las Metodologías De Análisis Forense Informático Para La Examinación De Datos En Medios Digitales. European Scientific Journa, 40-48.

13. Huamán, F. (2014). Diseño de procedimientos de auditoria de cumplimiento de la norma NTP - ISO/IEC 17799:2007 como parte del proceso de implementación de la norma técnica NTP-ISO/IEC 27001:2008 en instituciones del Estado Perano.

14. Ibarra, A., Mballa, L., Ibarra, M., \& Vega, M. (2019). Hacia Una Reconceptualización Teórica Y Metodológica Del Gobierno Abierto: Paradigma Explicativo De La Gobernabilidad Local. European Scientific Journal, 1-24.

15. Ibujés, J., \& Franco, A. (2019). Use of ICT and its relationship with the Objectives of Sustainable Development in Ecuador. RETOS. Revista de Ciencias de la Administración y Economía, 9(17), 37-53. doi:http://dx.doi.org/10.17163/ret.n17.2019.03

16. Kaplan, R., \& Norton, D. (1992). "The balanced scorecard- Measures that drive performance". Harvard Business Review.

17. Keupp, M., Palmié, M., \& Gassmann, O. (2012). The strategic management of innovation: A systematic review and paths for future 
research. International Journal of Management Reviews, 14(4), 367390.

18. López, A. (2014). El portal de ISO 27001 en español. Obtenido de http://www.iso27000.es/sgsi.html

19. Mahdzur, S., \& Salim, J. (2015). INFORMATION SYSTEMS INTEGRATION FACTORS IN ORGANIZATION: TOWARDS GOVERNMENT INFORMATION SYSTEMS SUSTAINABILITY. Journal of Theoretical \& Applied Information Technology, 71(2). Obtenido de http://www.jatit.org/volumes/Vol71No2/9Vol71No2.pdf

20. Marulanda, C., Valencia, F., \& López, M. (2017). Gobierno y gestión de TI en las entidades públicas de Manizales. Colombia. Administer, $1-21$.

21. Moon, H., Rugman, A., \& Verbeke, A. (1995). The generalized doble diamond approach to international competitiveness. Research in Global Strategic Management: A Research Annual, 97-114.

22. Moreira, T., Romero, S., Alava, L., Alcívar, E., \& Tarazona, K. (2018). El cambio de la matriz productiva y las nuevas tecnologías. Yachana Revista Científica, 5(3), 113-120.

23. Velásquez, T., Pérez, Y., \& Flórez, I. (2018). Adopción de prácticas de Gobierno de TI en la oficina de Admisiones, Registro y Control UFPSO. REVISTA COLOMBIANA DE TECNOLOGIAS DE AVANZADA (RCTA). doi:DOI: https://doi.org/10.24054/16927257.v31.n31.2018.2758 